\title{
SOME TYPES OF RELATIVE PARACOMPACTNESS
}

\author{
Vladimir Pavlović ${ }^{*}$
}

\begin{abstract}
This paper is a continuation of the study of relative topological properties. We use a characterization of paracompactness via a certain selection principle to introduce five types of relative paracompactness, provide examples showing that none of them coincide with each other and establish some results concerning finite unions of subspaces which are relatively paracompact in one or another of the defined senses.
\end{abstract}

\section{Introduction}

If $X$ is a topological space and $Y$ a subspace of $X$, then the properties of $Y$ in general will depend on the way in which that subspace is "placed" in $X$. Conversely, it is not rarely the case that having a subspace of a certain type placed in a particular way can largely effect the properties of the whole space. This suggests that to each topological property $\mathcal{P}$ a kind of its "relative" version can be assigned, now viewed on the family of all subspaces of a space $X$, in attempt to grasp one aspect of the fact how a certain subspace can be placed in $X$. In that sense, we talk about "a subspace $Y$ being $\mathcal{P}$-placed in $X$ ", or about " $Y$ relatively having the property $\mathcal{P}$ in $X$ ". Thus, we say that $Y$ is relatively Haussdorff in $X$ (see [3]) if for each pair of distinct points $x, y \in Y$ there exist two disjoint open in $X$ sets $U, V$ such that $x \in U$ and $y \in V$; according to Ju. Smirnov (see [5]) a subspace $Y$ of $X$ is normally placed in $X$ if for each open in $X$ set $U$ containing $Y$ there is a $F_{\sigma}$-set $Z \subseteq X$ such that $U \subseteq Z \subseteq X$; S. Mrówka (see [5]) calls $Y$ regularly placed in $X$ if for each point $x \in X \backslash Y$ there is a $F_{\sigma}$-set $Z \subseteq X$ such that $Y \subseteq Z \subseteq X \backslash\{x\}$ etc.

AMS (MOS) Subject Classification 1991. Primary: 54B05, 54D20.

Key words and phrases: Locally finite family, point finite family, open cover, relative paracompactness, $\mathrm{S}(\mathcal{O}, \mathcal{O})_{l f}, \mathrm{~S}(\mathcal{O}, \mathcal{O})_{p f}$.

${ }^{*}$ Supported by MNTR of Serbia, grant $\mathrm{N}^{0} 1233$. 
Relative properties have already been considered by many authors: relative compactness, countable compactness, relative dimensions, relative extremal disconnectedness, relative $G_{\delta}$-diagonal, relative cardinal invariants in $C_{p}$ theory and so on. Let us only mention the following result ( [5], pages 305 and 306): A Tychonoff space $X$ is Lindelöf (realcompact) if and only if $X$ is normally (regularly) placed in $\beta X$. As opposed to the relative ones the classical topological properties will be referred to as absolute properties. Let us mention here that a systematic study of relative topological properties was started by A.V. Arhangel'skii in [3] and continued later in a series of its papers (see for example [1], [2]).

In this paper we will be concerned with some relative versions of paracompactness, but in distinction from A.V. Arhangel'skii who has already considered this relative topological property defining it starting from the usual definition of paracompactness, we take another characterization of paracompactness as a base for deriving five relative types of it (actually four because it turns out that one of the defined versions coincides with the absolute paracompactness of the subspace under consideration).

Let us first establish some terminology and notation. For any sets $x, y$ the symbol $x \preceq y$ means $\forall z \in x \exists t \in y(z \subseteq t)$. $\mathcal{P}(X)$ denotes the set of all subsets of $x$. If $g$ is a function we use the symbol $g_{\rightarrow} A\left(g^{\leftarrow} A\right)$ to denote the (inverse) image of $A$ under $g$ and write $\operatorname{ran}(g)$ for the range of $g$. When we say that $x$ is point finite (point countable) on $y$ we mean that for each $b \in y$ the set $\{a \in x: b \in a\}$ is finite (countable). When a set $X$ is looked at as a topological space the corresponding topology will be denoted by $\mathcal{T}_{X}$. If $X$ is a space then $\mathcal{O}_{X}$ or just $\mathcal{O}$ (when there is no confusion to which $X$ the notation refers) stands for the family of all open covers of $X$.

Definicija 1.1. ( [4]) Let a space $X$, a subspace $Y$ of $X$ and $\mathcal{A}, \mathcal{B} \subseteq$ $\mathcal{P}(\mathcal{P}(X))$ be given. The notation $S(\mathcal{A}, \mathcal{B} ; X, Y)_{l f}\left(S(\mathcal{A}, \mathcal{B} ; X, Y)_{p f}\right)$ stands for the following statement:

For each sequence $\left\langle\mathcal{U}_{n}: n<\infty\right\rangle$ of elements of $\mathcal{A}$ there is a sequence $\left\langle\mathcal{V}_{n}: n<\infty\right\rangle$ of subsets of $\mathcal{P}(X)$ such that

- for each $n \mathcal{V}_{n} \preceq \mathcal{U}_{n}$, and the family $\mathcal{V}_{n}$ is locally finite on $Y$ with respect to the topology of $X$ ( point finite on $Y$ ),

$-\bigcup\left\{\mathcal{V}_{n}: n<\infty\right\} \in \mathcal{B}$.

Also we introduce the abbreviation $S(\mathcal{A}, \mathcal{B})_{l f} \stackrel{\text { def }}{=} S(\mathcal{A}, \mathcal{B} ; X, X)_{l f}$, and similarly for $S(\mathcal{A}, \mathcal{B})_{p f}$.

The notion we have just defined, for a convenient choice of the families $\mathcal{A}$ and $\mathcal{B}$, coincides exactly with the notion of paracompactness. More precisely, the following theorem holds (see [4]). 
Theorem 1. A regular space $X$ is paracompact if and only if $S(\mathcal{O}, \mathcal{O})_{l f}$ holds.

Thus, as we are interested in relative paracompactness we will primarily be concerned with the $S_{l f}$ principle. The main reason we consider the second one $\left(S_{p f}\right)$ is that most of the theorems in the paper are valid for it too, with almost identical (or even easier) proofs.

Now we can give an alternative (to that of Arhangel'skii) definition of relative paracompactness. We simply relativize the principle $S(\mathcal{O}, \mathcal{O})_{l f}$.

Definicija 1.2. Let a space $X$ and its subspace $Y$ be given. Put $\mathcal{O}_{X}(Y)=$ $\left\{\mathcal{B} \subseteq \mathcal{T}_{X}: Y \subseteq \bigcup \mathcal{B}\right\}$ and denote with $i-(Y \mid X)_{l f}\left(i-(Y \mid X)_{p f}\right)$ the fact $S(\mathcal{A}, \mathcal{B} ; X, Y)_{l f}\left(S(\mathcal{A}, \mathcal{B} ; X, Y)_{p f}\right)$, where:

for $i=1: \mathcal{A}=\mathcal{O}_{X}(Y), \mathcal{B}=\mathcal{O}_{X}(Y)$,

for $i=2: \mathcal{A}=\mathcal{O}_{X}(Y), \mathcal{B}=\mathcal{O}_{Y}$,

for $i=3: \mathcal{A}=\mathcal{O}_{X}, \mathcal{B}=\mathcal{O}_{X}$,

for $i=4: \mathcal{A}=\mathcal{O}_{X}, \mathcal{B}=\mathcal{O}_{X}(Y)$,

for $i=5: \mathcal{A}=\mathcal{O}_{X}, \mathcal{B}=\mathcal{O}_{Y}$.

If $i-(Y \mid X)_{l f}\left(i-(Y \mid X)_{p f}\right)$ holds we shall say that $Y$ is a $i-l f(i-p f)$ subspace of $X$.

So, there are five (potentially different) relative variants of paracompactness to be considered.

A direct consequence of the preceding definition is the following proposition, in which some basic relations between this $S_{l f}-i(i=\overline{1,5})$ relative properties are established.

Proposition 1. For a space $X$ and its subspace $Y$ the following implications hold:

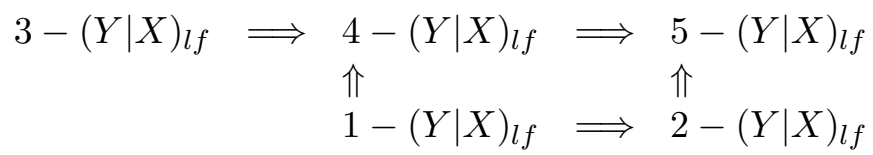

If $X$ is compact then $3-(Y \mid X)_{l f}$ for any subspace $Y$ of $X$. Also, if $Y$ is compact then $1-(Y \mid X)_{l f}$ for any $X$ containing $Y$.

Now we list several easy facts about the $S_{l f}-i$ properties.

Proposition 2. For a space $X$ and $Y \subseteq X$ the following statements are true:

1) $4-(Y \mid X)_{p f} \Leftrightarrow 5-(Y \mid X)_{p f} i 1-(Y \mid X)_{p f} \Leftrightarrow 2-(Y \mid X)_{p f}$;

2) if $\bar{Y}=X$ then $4-(Y \mid X)_{l f} \Leftrightarrow 5-(Y \mid X)_{l f} i 1-(Y \mid X)_{l f} \Leftrightarrow 2-$ $(Y \mid X)_{l f}$ 
3) if $Y=\bar{Y}$ then $1-(Y \mid X)_{a b} \Leftrightarrow 4-(Y \mid X)_{a b}, 2-(Y \mid X)_{a b} \Leftrightarrow 5-(Y \mid X)_{a b}$, where "ab" replaces any of the notations "lf" or "pf", as well as $3-(Y \mid X)_{p f} \Leftrightarrow 4-(Y \mid X)_{p f} ;$

4) if $X$ is a perfectly normal space and $Y=\bar{Y}$, then $3-(Y \mid X)_{l f} \Leftrightarrow$ $4-(Y \mid X)_{l f}$

5) $Y \in S\left(\mathcal{O}_{Y}, \mathcal{O}_{Y}\right)_{a b} \Leftrightarrow 2-(Y \mid X)_{a b}$, where "ab" replaces any of the notations "lf" or "pf";

6) $X \in S(\mathcal{O}, \mathcal{O})_{a b} \Rightarrow 3-(Y \mid X)_{a b}$, where "ab" stands for either "lf" or "pf";

7) if $Y=\bar{Y}$ then $X \in S(\mathcal{O}, \mathcal{O})_{a b} \Rightarrow 1-(Y \mid X)_{a b}$, with "ab" as before;

8) if $Z \subseteq Y$ then $i-(Y \mid X)_{a b} \Rightarrow i-(Z \mid X)_{a b}$, for $i \in\{3,4,5\}$ and "ab" as before;

9) if $Z \subseteq Y=\bar{Y}$ then $i-(Z \mid X)_{a b} \Rightarrow i-(Z \mid Y)_{a b}$, for $i \in\{3,4,5\}$ and "ab" as above.

Proof. The statements under 1) and 2) follow directly from the next few easy observations:

Let $\mathcal{L} \subseteq \mathcal{T}_{Y}, \mathcal{C} \subseteq \mathcal{T}_{X}$ and $\mathcal{L} \preceq \mathcal{C}$. For each $A \in \mathcal{L}$ take a $U_{A} \in \mathcal{C}$ and a $V_{A} \in \mathcal{T}_{X}$, such that $A=Y \cap V_{A}$ and $A \subseteq U_{A}$. Put $\mathcal{L}^{\prime}=\left\{U_{A} \cap V_{A}: A \in \mathcal{L}\right\}$. Clearly, $\mathcal{L} \preceq \mathcal{L}^{\prime} \preceq \mathcal{C}$ and $\mathcal{L}^{\prime} \subseteq \mathcal{T}_{X}$. Then for each $y \in Y$ and each $A \in \mathcal{L}$ we must have $y \in A \Leftrightarrow y \in U_{A} \cap V_{A}$. Also, if $Y=\bar{X}$, then for each $y \in Y, G \in \mathcal{T}_{X}$ and $A \in \mathcal{L}$, where $y \in G$, we have that $A \cap G=\emptyset \Leftrightarrow\left(U_{A} \cap V_{A}\right) \cap G=\emptyset$. Consequently, if $\mathcal{L}$ is point-finite on $Y$ so is $\mathcal{L}^{\prime}$, and if addition $Y=\bar{Y}$, then if $\mathcal{L}$ is locally finite on $Y$ so is $\mathcal{L}^{\prime}$. Finally, $\bigcup \mathcal{L} \subseteq \bigcup \mathcal{L}^{\prime}$.

Now let us prove the claim stated under 4$)$. Let $4-(Y \mid X)_{l f}$, where $Y=\bar{Y}$ and let a sequence of open covers of $X\left\langle\mathcal{U}_{n}: n<\infty\right\rangle$ be given. Applying $4-(Y \mid X)_{l f}$ to that sequence we obtain another sequence $\left\langle\mathcal{V}_{n}: n<\infty\right\rangle$, where $\mathcal{V}_{n} \subseteq \mathcal{T}_{X}$, such that $\mathcal{V}_{n} \preceq \mathcal{U}_{n}$, each $\mathcal{V}_{n}$ a family locally finite on $Y$, and $Y \subseteq \bigcup \bigcup\left\{\mathcal{V}_{n}: n<\infty\right\}$. As $X$ is perfectly normal and $Y$ closed in $X$, there is a sequence of open sets $\left\langle U_{n}: n<\infty\right\rangle$ such that $Y=\bigcap_{n<\infty} U_{n}=\bigcap_{n<\infty} \overline{U_{n}}$. Put $\mathcal{U}_{n}^{\prime}=\left\{U \cap\left(X \backslash \overline{U_{n}}\right): U \in \mathcal{U}_{n}\right\}$. Then, for $\mathcal{M}_{n}=\mathcal{V}_{n} \cup \mathcal{U}_{n}^{\prime} \subseteq \mathcal{T}_{X}$, we have that $\mathcal{M}_{n} \preceq \mathcal{U}_{n}$, each of the families $\mathcal{M}_{n}$ is locally finite on $Y$, and $\bigcup\left\{\mathcal{M}_{n}: n<\infty\right\}$ is an open cover of $X$.

The remaining statements of the proposition are trivially established.

Apparently, the relation $2-(Y \mid X)_{l f}$, i. e. $2-(Y \mid X)_{p f}$ does not depend on the particular way in which $Y$ is placed in $X$, it actually describes the absolute paracompactness of $Y$.

In the next two examples $\mathbf{R}$ will denote the set of reals. Also, $(a, b)$ will stand both for the appropriate ordered pair and for the set $\{x \in \mathbf{R}: a<$ 
$x<b\}$, in which case, of course, $a, b \in \mathbf{R}$. It will be clear from the context which one is the case. $[a, b]$, where $a, b \in \mathbf{R}$, means as usual the closed segment $\{x \in \mathbf{R}: a \leq x \leq b\}$ of the real line.

Example 1. Let $X$ be the Niemytzky plane [5]: the supporting set is $X=\left\{(x, y) \in \mathbf{R}^{2}: y>0\right\} \cup \mathbf{R}$, and it is topologized as follows: if $(a, b) \in$ $X \backslash \mathbf{R}$ then the family of all the sets of the form $K(a, b ; r)=\{(x, y) \in$ $\left.X: \sqrt{(x-a)^{2}+(y-b)^{2}}<r\right\}$, where $r>0$ is an arbitrary real number, constitutes an open neighborhood base at that point; if $a \in \mathbf{R}$ then an open neighborhood base at $a$ is given by the family of all $B(a, b)=K(a, b ; b) \cup\{a\}$, where $b>0$ is an arbitrary real number.

It is not difficult to see that $\mathbf{R} \subseteq X$ is a discrete subspace of $X$. Therefore it is paracompact, so $2-(\mathbf{R} \mid X)_{l f}$. Let us show, on the other hand, that $1-(\mathbf{R} \mid X)_{l f}$ does not hold.

Assume to the contrary that $\mathbf{R}$ is a $1-l f$ subspace of $X$ and consider for each $n$ a family $\mathcal{A}_{n}=\mathcal{A}=\{B(x, 1): x \in \mathbf{R}\}$ of open subsets of $X$ covering the subspace $\mathbf{R}$. Then there is a sequence of families $\mathcal{L}_{n}$ of open subsets of $X$ such that $\bigcup_{n<\infty} \mathcal{L}_{n} \supseteq \mathbf{R}$, and such that, for each $n, \mathcal{L}_{n} \preceq \mathcal{A}_{n} \equiv \mathcal{A}$ and $\mathcal{L}_{n}$ is locally finite on $\mathbf{R}$.

For each $x \in \mathbf{R}$ there is a $n_{x}$ and a $U_{x} \in \mathcal{L}_{n_{x}}$ such that $x \in U_{x}$.

On the other hand, as $\mathcal{L}_{n_{x}} \preceq \mathcal{A}$, there is a $y \in \mathbf{R}$ such that $U_{x} \subseteq B(y, 1)$. From $\{y\}=\mathbf{R} \cap B(y, 1) \supseteq \mathbf{R} \cap U_{x} \supseteq\{x\}$ it follows $y=x$ and $\{x\}=\mathbf{R} \cap U_{x}$. Since $U_{x}$ is open, there is a real number $\varepsilon_{x}>0$ such that $x \in B\left(x, \varepsilon_{x}\right) \subseteq U_{x}$. So:

$$
\begin{gathered}
\{x\}=\mathbf{R} \cap B\left(x, \varepsilon_{x}\right)=\mathbf{R} \cap U_{x} ; \\
x \in B\left(x, \varepsilon_{x}\right) \subseteq U_{x} ; K\left(x, \varepsilon_{x} ; \varepsilon_{x}\right) \subseteq B\left(x, \varepsilon_{x}\right) \\
x \neq y \Rightarrow U_{x} \neq U_{y} .
\end{gathered}
$$

Put $S_{n}=\left\{x \in \mathbf{R}: n_{x}=n\right\}$. For each $n \in \mathbf{N}$, and each real number $a>0$, let us call a closed segment $I$ of the real line " $(n, a)$ - good" if $\forall x \in$ $S_{n} \cap I\left(\varepsilon_{x}<a\right)$.

Claim. For each $m \in \mathbf{N}$, a real number $\theta>0$ and a closed segment of the real line $[p, q]$, with $p<q$, there is a $(m, \theta)$-good closed segment $I \subseteq[p, q] \ldots(*)$.

\section{Indeed:}

Let us first note that $\forall x \in S_{m}\left(U_{x} \in \mathcal{L}_{m}\right)$. Fix a $a \in(p, q)$. $\mathcal{L}_{m}$ is locally finite on the set $\mathbf{R}$ so there must be a real number $r>0$ and a finite set $\left\{V_{1}, \ldots, V_{k}\right\} \subseteq \mathcal{L}_{m}$ such that $\forall V \in \mathcal{L}_{m} \backslash\left\{V_{1}, \ldots, V_{k}\right\}(V \cap B(a, r)=\emptyset)$. By (2), this implies that there must be a finite set $\left\{x_{1} \ldots, x_{k}\right\} \subseteq \mathbf{R}$ such that 
$\forall x \in S_{m}\left(x \notin\left\{x_{1}, \ldots, x_{k}\right\} \Rightarrow U_{x} \cap B(a, r)=\emptyset\right)$. So, having in mind (1) as well as $K(a, r ; r) \subseteq B(a, r)$ :

$$
\forall x \in S_{m} \backslash\left\{x_{1}, \ldots, x_{k}\right\}\left(K\left(x, \varepsilon_{x} ; \varepsilon_{x}\right) \cap K(a, r ; r)=\emptyset\right) .
$$

Consequently, for $x \in S_{m}$ with $x \neq x_{i}$ we must have:

$$
\varepsilon_{x}+r \leq \sqrt{(x-a)^{2}+\left(r-\varepsilon_{x}\right)^{2}} \leq|x-a|+\left|r-\varepsilon_{x}\right| .
$$

Let $n>0$ be any integer such that $\frac{1}{n}<\min (2 r, \theta),\left(\left(a-\frac{1}{n}, a+\frac{1}{n}\right) \backslash\{a\}\right) \cap$ $\left\{x_{1}, \ldots, x_{k}\right\}=\emptyset$ and $\left(a-\frac{1}{n}, a+\frac{1}{n}\right) \subseteq[$ 品 $[p, q]$. Then, according to $(4)$, for each $x \in S_{m} \cap\left(\left(a-\frac{1}{n}, a+\frac{1}{n}\right) \backslash\{a\}\right)$ we have that:

$$
\varepsilon_{x}+r \leq \frac{1}{n}+\left|r-\varepsilon_{x}\right|
$$

and

$\theta>\frac{1}{n} \geq \varepsilon_{x}+r-\left|r-\varepsilon_{x}\right|= \begin{cases}\varepsilon_{x}+r-r+\varepsilon_{x}=2 \varepsilon_{x} & \\ \varepsilon_{x}+r+r-\varepsilon_{x}=2 r, & \text { which is impossible } \\ & \text { because } \frac{1}{n}<2 r .\end{cases}$

In other words, we conclude that each $x \in S_{m} \cap\left(\left(a-\frac{1}{n}, a+\frac{1}{n}\right) \backslash\{a\}\right)$ must satisfy: $2 \varepsilon_{x}<\theta$, implying that any closed segment $I \subseteq\left(a-\frac{1}{n}, a\right)$ is $(m, \theta)$ - good, which, together with $I \subseteq[p, q]$, proves $(*)$.

Now let, according to $(*), I_{1} \subseteq[0,1]$ be a $(1,1)$ - good segment (of length not greater than 1 ). If the segment $I_{n}$ has been constructed so that it is $\left(i, \frac{1}{n}\right)$ - good for each $i \in\{1, \ldots, n\}$ and with length not greater than $\frac{1}{n}$, choose a segment $J_{n} \subseteq I_{n}$ of length not greater than $\frac{1}{n+1}$, as well as segments $J_{n} \supseteq I_{1}^{n+1} \supseteq I_{2}^{n+1} \supseteq \cdots \supseteq I_{n+1}^{n+1}$, such that for each $i \in\{1, \ldots, n+1\}$ $I_{i}^{n+1}$ is $\left(i, \frac{1}{n+1}\right)-\operatorname{good}$ and put $I_{n+1}=I_{n+1}^{n+1}$. Since $I_{n+1} \subseteq I_{i}^{n+1}$ and $I_{i}^{n+1}$ is $\left(i, \frac{1}{n+1}\right)$ - good, the segment $I_{n+1}$ must also be $\left(i, \frac{1}{n+1}\right)$ - good for each $i \in\{1, \ldots, n+1\}$. Finally, $I_{n+1} \subseteq I_{n}$ and the length of $I_{n+1}$ is not greater than $\frac{1}{n+1}$.

So, we have constructed a decreasing sequence $\left\langle I_{n}: n<\infty\right\rangle$ of closed intervals with lengths converging to 0 such that for all $n, m$ with $n \leq m I_{m}$ is $\left(n, \frac{1}{m}\right)$ - good. As there must be an $x \in \bigcap_{n<\infty} I_{n}$, and as every $I_{m}$ is $\left(n_{x}, \frac{1}{m}\right)$ good for $m \geq n_{x}$, it must be that $\varepsilon_{x}<\frac{1}{m}$ for all $m \geq n_{x}$, i. e. $\varepsilon_{x}=0$, which is impossible.

According to Proposition 2. under (3, this is also an example of a pair $Y, X$ with $5-(Y \mid X)_{l f}$ but not $4-(Y \mid X)_{l f}$.

Example 2. Let $X$ denote the same space as in the example above and fix an arbitrary compact $K$ containing the set $\mathbf{R}$ as its discrete subspace. Call a pair $(U, V) \in \mathcal{T}_{X} \times \mathcal{T}_{K}$ a $l$-pair if $U \cap \mathbf{R}=V \cap \mathbf{R}$. Put $Y=X \cup K$, and define the following (Hausdorff) topology on the set $Y: \mathcal{T}_{Y}=\{U \cup V:(U, V)$ je $l$-par $\}$. Let us prove $4-(\mathbf{R} \mid Y)_{l f}$. 
Let $\mathcal{A} \in \mathcal{O}_{Y}$. Consider $\mathcal{A}_{1}=\left\{V \in \mathcal{T}_{K}: \exists U \in \mathcal{T}_{X}((U, V)\right.$ is l-pair; $U \cup$ $V \in \mathcal{A}\}$ and fix a function $f: \mathcal{A}_{1} \rightarrow \mathcal{T}_{X}$ such that for each $V \in \mathcal{A}_{1}(f(V), V)$ is a $l$-pair and $f(V) \cup V \in \mathcal{A}$. As $\mathcal{A}_{1} \in \mathcal{O}_{K}$ and as $K$ is compact there are $V_{1}, \ldots, V_{n} \in \mathcal{A}_{1}$ such that $K=\bigcup_{i=1}^{n} V_{i}$. Then $\mathbf{R} \subseteq \bigcup_{i=1}^{n}\left(f\left(V_{i}\right) \cup V_{i}\right)$, where, of course, for each $i=1, \ldots, n, f\left(V_{i}\right) \cup V_{i} \in \mathcal{A}$, and the family $\left\{f\left(V_{i}\right) \cup V_{i}\right.$ : $i=1 \ldots, n\}$ is finite (whence also locally finite at every point of the space $Y)$. So obviously $4-(\mathbf{R} \mid Y)_{l f}$.

On the other hand, it cannot be $1-(\mathbf{R} \mid Y)_{l f}$ : Since $\mathbf{R}$ is a discrete subspace of $K$ we can, for each $x \in \mathbf{R}$, choose a $V_{x} \in \mathcal{T}_{K}$ such that $\{x\}=$ $V_{x} \cap \mathbf{R}$. Putting, for each $n \in \mathbf{N}, \mathcal{L}_{n}=\left\{B(x, 1) \cup V_{x}: x \in \mathbf{R}\right\}$ (where $B(x, \varepsilon)$ stands for the same thing as before) and reasoning in the same manner as in the preceding example it is not difficult to see that there can be no sequence $\left\langle\mathcal{P}_{n}: n<\infty\right\rangle$ with $\mathcal{P}_{n} \subseteq \mathcal{T}_{Y}$ for each $n \in \mathbf{N}$, such that $\mathbf{R} \subseteq \bigcup \bigcup\left\{\mathcal{P}_{n}: n<\infty\right\}$ and for each $n$ the family $\mathcal{P}_{n}$ is locally finite on $\mathbf{R}$ as well as $\mathcal{P}_{n} \preceq \mathcal{L}_{n}$.

Example 3. If $X$ is compact and $Y$ any non paracompact subspace of $X$ then $i-(Y \mid X)_{l f}$ for $i \in\{3,4,5\}$, but not $2-(Y \mid X)_{l f}$ (hence neither $\left.1-(Y \mid X)_{l f}\right)$.

Example 4. If $Y$ is a countable subspace of $X$ then trivially $1-$ $(Y \mid X)_{l f}$ (so $4-(Y \mid X)_{l f}$ too) holds. If in addition $X$ is not Lindëlof and $\bar{Y}=X$, then it cannot be $3-(Y \mid X)_{l f}$. This is because any family of open sets of a space which is locally finite on a dense countable subspace $S$ of it (even point countable on $S$ ) must be countable. Thus the assertion follows if we choose any open cover $\mathcal{K}$ of $X$ with no countable subcover and take all the $\mathcal{U}_{n}$-s in the definition of $3-(Y \mid X)_{l f}$ (see Definition 1.) to be $\mathcal{K}$.

So, any non Lindëlof separable space (e.g. the Niemitcky plane) is an example showing that neither $1-(Y \mid X)_{l f}$ nor $4-(Y \mid X)_{l f}$ imply $3-(Y \mid X)_{l f}$.

In the next few theorems we will try to answer the question when a certain type of relative paracompactness of two (finitely many) subspaces implies the same property of their union.

A trivial, but useful observation is formulated as follows.

Lemma 1. If $\mathcal{K}$ is an arbitrary locally finite (point-finite) on $S$ family of subsets of the space $X$, where $S \subseteq X$, and $\mathcal{L}$ any partition of the set $\mathcal{K}(i$. e. $\bigcup \mathcal{L}=\mathcal{K} i \forall x, y \in \mathcal{L}(x \neq y \Rightarrow x \cap y=\emptyset))$, then the family $\{\bigcup x: x \in \mathcal{L}\}$ is also locally finite (point-finite) on $S$.

Proof. Elementary.

We shall often make use of this fact below.

A union of two closed $2-l f$ subspaces is again a $2-l f$ subspace. 
Theorem 2. If $F_{1}, F_{2}$ are both closed and paracompact subspaces of a space $X$ then the subspace $F_{1} \cup F_{2}$ is also paracompact.

Proof. Let an arbitrary family $\mathcal{U} \subseteq \mathcal{T}_{X}$ be given such that $F_{1} \cup F_{2} \subseteq$ $\cup \mathcal{U}$. As $F_{1}$ is paracompact there exists a $\mathcal{L} \subseteq \mathcal{T}_{F_{1}}$ such that $F_{1}=\bigcup \mathcal{L}$ and $\mathcal{L} \preceq \mathcal{U}$, and such that the family $\mathcal{L}$ is locally finite on $F_{1}$ (whence on the whole space $X$, because $F_{1}$ is closed). Take any function $f: \mathcal{L} \rightarrow \mathcal{T}_{X}$ such that $\forall U \in \mathcal{L}\left(U=F_{1} \cap f(U) \wedge \exists V \in \mathcal{U}(f(U) \subseteq V)\right)$. Let $\mathcal{L}^{\prime}=\left\{f(U): U^{\prime} \in \mathcal{L}\right\}$ i $\mathcal{U}^{\prime}=\left\{\left(X \backslash F_{1}\right) \cap U: U \in \mathcal{U}\right\}$.

$\mathcal{L}^{\prime} \cup \mathcal{U}^{\prime}$ is a family of sets open in $X$ which covers $F_{2}$ so, as $F_{2}$ is paracompact, there is a $\mathcal{V} \subseteq \mathcal{T}_{F_{2}}$, where $\mathcal{V} \preceq \mathcal{L}^{\prime} \cup \mathcal{U}^{\prime}, F_{2} \subseteq \cup \mathcal{V}$ such that the family $\mathcal{V}$ is locally finite on $F_{2}$. Put $\mathcal{V}_{1}^{\prime}=\{A \in \mathcal{V}: \exists U \in \mathcal{L}(A \subseteq f(U))\}$ and choose a $g: \mathcal{V}_{1}^{\prime} \rightarrow \mathcal{L}$ such that $\forall A \in \mathcal{V}_{1}^{\prime}(A \subseteq f(g(A))=h(A))$, where $h=f \circ g$. For a $A \in \mathcal{V}_{1}^{\prime}$ find a $O^{\prime}(A) \in \mathcal{T}_{X}$ such that $A=F_{2} \cap O^{\prime}(A)$ and denote $O(A)=$ $O^{\prime}(A) \cap h(A) \in \mathcal{T}_{X}$. For a $U \in \operatorname{ran}(g)$ let $\Theta(U)=\bigcup\left\{O(A): A \in g^{\leftarrow}\{U\}\right\} \in \mathcal{T}_{X}$ and $\Theta_{i}(U)=F_{i} \cap \Theta(U), \quad i=1,2$. So, $\Theta_{1}(U) \cup \Theta_{2}(U)=\Theta(U) \cap\left(F_{1} \cup F_{2}\right)$ is open in $F_{1} \cup F_{2}$. Finally, put $\mathcal{V}_{1}=\left\{\Theta_{1}(U) \cup \Theta_{2}(U): U \in \operatorname{ran}(g)\right\}$.

Clearly, from $\mathcal{L}^{\prime} \preceq \mathcal{U}$ it follows $\mathcal{V}_{1} \preceq \mathcal{U}$ (because $\Theta_{1}(U) \cup \Theta_{2}(U) \subseteq$ $\Theta(U)=\bigcup\{O(A): A \in g \rightarrow\{U\}\} \subseteq \bigcup\{f(g(A)): g(A)=U\} \subseteq f(U) \subseteq V$, for a $V \in \mathcal{U})$.

By construction we have that $\Theta_{1}(U) \subseteq U$ for each $U \in \operatorname{ran}(g) \subseteq \mathcal{L}$, so as $\mathcal{L}$ is locally finite on $X$ so is $\left\{\Theta_{1}(U): U \in \operatorname{ran}(g)\right\}$. Also, $\Theta_{2}(U)=$ $\bigcup g^{\leftarrow}\{U\}=\bigcup\left\{A \in \mathcal{V}_{1}^{\prime}: g(A)=U\right\}$, so by Lemma 1. (considering that $\mathcal{V}_{1}^{\prime} \subseteq \mathcal{V}$ is locally finite on $F_{2}$, hence on $X$ too) the family $\left\{\Theta_{2}(U): U \in \operatorname{ran}(g)\right\}$ is locally finite on $X$.

Therefore $\mathcal{V}_{1} \subseteq \mathcal{T}_{F_{1} \cup F_{2}}$ must be locally finite on $X$ too (equivalently: on $\left.F_{1} \cup F_{2}\right)$.

Put $\mathcal{V}_{2}=\left\{A \in \mathcal{V}: \exists U \in \mathcal{U}\left(A \subseteq\left(X \backslash F_{1}\right) \cap U\right)\right\} \preceq \mathcal{U}$. One readily sees that, for each $A \in \mathcal{V}_{2}$ we must have that $A=\left(X \backslash F_{1}\right) \cap U \cap F_{2}=$ $\left(\left(X \backslash F_{1}\right) \cap U\right) \cap\left(F_{1} \cup F_{2}\right)$, which implies that $\mathcal{V}_{2} \subseteq \mathcal{T}_{F_{1} \cup F_{2}}$. From $\mathcal{V}_{2} \subseteq \mathcal{V}$ it follows that $\mathcal{V}_{2}$ is locally finite on $F_{1} \cup F_{2}$.

Finally, it can easily be seen that $F_{2} \subseteq \bigcup\left(\mathcal{V}_{1} \cup \mathcal{V}_{2}\right)$.

Now, we have defined a family $\mathcal{A}=\mathcal{V}_{1} \cup \mathcal{V}_{2} \subseteq \mathcal{T}_{F_{1} \cup F_{2}}$ such that $\mathcal{A} \preceq \mathcal{U}$, $\mathcal{A}$ is locally finite on $F_{1} \cup F_{2}$, and $F_{2} \subseteq \cup \mathcal{A}$. Replacing the roles of the subspaces $F_{1}$ and $F_{2}$, in exactly the same way we can obtain a $\mathcal{B} \subseteq \mathcal{T}_{F_{1} \cup F_{2}}$ with the property that $\mathcal{B} \preceq \mathcal{U}, \mathcal{B}$ is locally finite on $F_{1} \cup F_{2}$ and $F_{1} \subseteq \cup \mathcal{B}$.

$\mathcal{A} \cup \mathcal{B}$ is the required family which is locally finite on $F_{1} \cup F_{2}$, refines $\mathcal{U}$, with $F_{1} \cup F_{2}=\bigcup(\mathcal{A} \cup \mathcal{B})$, and which witnesses for the paracompactness of the subspace $F_{1} \cup F_{2}$.

The analoguous property for " $3-l f$ " subspaces is established as shown below. 
Theorem 3. If $F_{1}, F_{2} \subseteq X$ are such that $3-\left(F_{i} \mid X\right)_{l f}, \quad i=1,2$, then $3-\left(F_{1} \cup F_{2} \mid X\right)_{l f}$.

Proof. Let a sequence $\left\langle\mathcal{U}_{k, i}: k, i<\infty\right\rangle$ be given such that $\mathcal{U}_{k, i} \subseteq \mathcal{T}_{X}$ and such that any of the families $\mathcal{U}_{k, i}$ covers $X$.

Apply the fact that $3-\left(F_{1} \mid X\right)_{l f}$ to each of the sequences $\left\langle\mathcal{U}_{k, i}: i<\infty\right\rangle$ in order to obtain for each $k \in \mathbf{N}$ a sequence $\left\langle\mathcal{L}_{k, i}: i<\infty\right\rangle$ such that $\mathcal{L}_{k, i} \subseteq \mathcal{T}_{X}$, families $\mathcal{L}_{k, i}$ are all locally finite on $F_{1}$, each of the sets $\bigcup_{i<\infty} \mathcal{L}_{k, i}$ covers $X$ and such that $\mathcal{L}_{k, i} \preceq \mathcal{U}_{k, i}$.

Now apply $3-\left(F_{2} \mid X\right)_{l f}$ to the sequence $\left\langle\bigcup_{i<\infty} \mathcal{L}_{k, i}: k<\infty\right\rangle$ so as to obtain a sequence $\left\langle\mathcal{J}_{k}: k<\infty\right\rangle$, where $\mathcal{J}_{k} \subseteq \mathcal{T}_{X}$, each $\mathcal{J}_{k}$ is locally finite on $F_{2}$, the family $\bigcup_{k} \mathcal{J}_{k}$ covers $X$ and $\mathcal{J}_{k} \preceq \bigcup_{i<\infty} \mathcal{L}_{k, i}$. For each $k, i$ put $\mathcal{J}_{k, i}^{\prime}=\left\{A \in \mathcal{J}_{k}: \exists U \in \mathcal{L}_{k, i}(A \subseteq U)\right\}$ and take any $g_{k, i}: \mathcal{J}_{k, i}^{\prime} \rightarrow \mathcal{L}_{k, i}$ such that $\forall A \in \mathcal{J}_{k, i}^{\prime}\left(A \subseteq g_{k, i}(A)\right)$. Denote $\mathcal{J}_{k, i}=\left\{\bigcup g_{k, i}^{\leftarrow}\{U\}: U \in \mathcal{L}_{k, i}\right\}$. Obviously: $\bigcup \mathcal{J}_{k, i}^{\prime} \stackrel{\mathcal{J}}{=} \bigcup \mathcal{J}_{k, i}, \mathcal{J}_{k, i} \preceq \mathcal{L}_{k, i} \preceq \mathcal{U}_{k, i}$. As the family $\mathcal{J}_{k, i}$ is of the form $\left\{V_{U}: U \in \mathcal{L}_{k, i}\right\}$, where $V_{U} \subseteq U$, and as $\mathcal{L}_{k, i}$ is locally finite on $F_{1}$, we conclude that $\mathcal{J}_{k, i}$ must also be locally finite on $F_{1}$. The family $\mathcal{J}_{k, i}^{\prime}$ is locally finite on $F_{2}$ (because such is $\mathcal{J}_{k}$ ), so (in view of Lemma 1 . as well as the way in which we constructed the $\mathcal{J}_{k, i}$-s) the family $\mathcal{J}_{k, i}$ is also locally finite on $F_{2}$. And so, we have defined the families $\mathcal{J}_{k, i} \prec \mathcal{U}_{k, i}$ of open sets each of which is locally finite on $F_{1} \cup F_{2}$.

Since $\mathcal{J}_{k} \preceq \bigcup_{i<\infty} \mathcal{L}_{k, i}$ it must be $\mathcal{J}_{k}=\bigcup_{i<\infty} \mathcal{J}_{k, i}^{\prime}$, and therefore:

$$
\bigcup \mathcal{J}_{k}=\bigcup \bigcup_{i<\infty} \mathcal{J}_{k, i}^{\prime}=\bigcup \bigcup \mathcal{J}_{k, i}^{\prime}=\bigcup \bigcup \mathcal{J}_{k, i}=\bigcup \bigcup_{i<\infty} \mathcal{J}_{k, i}
$$

So

$$
X=\bigcup \bigcup_{k<\infty} \mathcal{J}_{k}=\bigcup \bigcup \bigcup_{k<\infty} \mathcal{J}_{k, i}=\bigcup \bigcup \bigcup_{k<\infty} \bigcup_{i<\infty} \mathcal{J}_{k, i}=\bigcup \bigcup_{k, i<\infty} \mathcal{J}_{k, i}
$$

i. e. $\bigcup_{k, i<\infty} \mathcal{J}_{k, i}$ is a cover of $X$.

And so, the sequence $\left\langle\mathcal{J}_{k, i}: k, i<\infty\right\rangle$ proves $3-\left(F_{1} \cup F_{2} \mid X\right)_{l f}$.

Practically repeating the proof of the previous theorem we obtain the next one.

Theorem 4. If $F_{1}, F_{2} \subseteq X$ are such that $3-\left(F_{i} \mid X\right)_{p f}, \quad i=1,2$, then $3-\left(F_{1} \cup F_{2} \mid X\right)_{p f}$.

And finally Theorems 4.,3. and Proposition 2. (under 3) and 4) ) give us the next two observations.

Theorem 5. If $F_{1}, F_{2}$ are both closed subspaces of a space $X$ with $4-$ $\left(F_{i} \mid X\right)_{p f}, \quad i=1,2$, then $4-\left(F_{1} \cup F_{2} \mid X\right)_{p f}$.

Theorem 6. If $F_{1}, F_{2}$ are both closed subspaces of a perfectly normal space $X$ with $4-\left(F_{i} \mid X\right)_{l f}, \quad i=1,2$ then also $4-\left(F_{1} \cup F_{2} \mid X\right)_{l f}$. 


\section{References}

[1] A.V. Arhangel'skii, From classic topological invariants to relative topological properties, (preprint).

[2] A.V. Arhangel'skii, Relative topological properties and relative topological spaces, Topology Appl. 20 (1996), 1-13.

[3] A.V. Arhangel'skii and H.M.M. Genedi, Beginnings of the theory of relative topological properties, In: General Topology. Spaces and Mappings, MGU, Moscow, 1989, 3-48.

[4] L. Babinkostova, Lj.D.R. Kočinac and M. Scheepers, Notes on selection principles in Topology (I): Paracompactness, (preprint).

[5] R. Engelking, General Topology, PWN, Warszawa, 1977.

Faculty of Sciences and Mathematics University of Niš Višegradska 33, $18000 \mathrm{Niš}$

Serbia \& Montenegro (Yugoslavia) 